\title{
Proteomics and Transcriptomics Investigation on longissimus Muscles in Large White and Casertana Pig Breeds
}

\author{
Leonardo Murgiano, ${ }^{\dagger}$ Angelo D’Alessandro, ${ }^{\ddagger}$ Maria Giulia Egidi, ${ }^{\ddagger}$ Alessandra Crisà, ${ }^{\dagger}$ \\ Gianluca Prosperini, ${ }^{\dagger}$ Anna Maria Timperio, ${ }^{\ddagger}$ Alessio Valentini, ${ }^{\dagger}$ and Lello Zolla*, ${ }^{*}$ \\ Dipartimento di Produzioni Animali, Università della Tuscia, Via de Lellis, 01100 Viterbo, Italy, and \\ Dipartimento di Scienze Ambientali, Università della Tuscia, L.go dell'Università snc, 01100, Viterbo, Italy
}

Received July 6, 2010

\begin{abstract}
Consumer complaints against the blandness of modern lean meat and the frequent reference to the more strongly flavored meat that was available years ago have prompted reconsideration of high fatdepositing typical pig breeds. Casertana and Large White pig breeds are characterized by a different tendency toward fat accumulation as they exhibit opposite genetic and physiological traits with respect to the energy metabolism. These physiological differences were investigated in longissimus lumborum muscles through proteomics (2-DE, MS/MS) and microarray approaches. Data were analyzed for pathway and network analyses, as well as GO term enrichment of biological functions. As a result, Casertana showed a greater amount of proteins involved in glycolitic metabolism and mainly rely on fast-mobilizable energy sources. Large White overexpressed cell cycle and skeletal muscle growth related genes. Metabolic behavior and other implications are discussed.
\end{abstract}

Keywords: Casertana pig breed • Large White pig breed • fat deposition • interactomics • proteomics - microarray

\section{Introduction}

Europe and China are the origin of about $70 \%$ of breed diversity in the world. ${ }^{1}$ In both China and Europe, pig has been and remains a major meat producer. Molecular approaches to improve pig meat quality have been rapidly emerging and gaining momentum, ${ }^{2}$ especially those kinds of investigations that are oriented toward comparisons between different breeds through integrated proteomics and transcriptomics analyses. ${ }^{3,4}$

Europe has 228 listed existing breeds, plus 105 now extinct; China has 118 listed breeds and 10 more extinct as it has been reported in the World Watch List for Domestic Animal Diversity. ${ }^{5}$ In the late 18th century, many new pig breeds, such as the Large White (LW) and the Berkshire in the U.K., came into existence and were distributed globally to replace or improve local breeds. ${ }^{6}$ Indeed, many of the European local breeds have been heavily altered and three-quarters of local or traditional breeds are extinct or marginalized. This is the case of the black pigs of Italy, which have been long questioned to be influenced by pigs imported from China-although it is still controversial, as they appear to be closely related to the Iberian groups ${ }^{1}$ or rather belong to an independent area of domestication on the Italian peninsula, which is opposed to the rest of Europe. ${ }^{7}$ Italian black pigs might date back to Roman times or, more likely, to the 17 th century. ${ }^{8}$ One Italian breed in particular, the Neapolitan, played a pivotal role in the formation of some of

* To whom correspondence should be addressed. Lello Zolla. E-mail: zolla@unitus.it. Tel.: +390761 357100. Fax: +39 0761 357179. L.go dell'Università snc, University of Tuscia, 01100 Viterbo (VT), Italy.

$\dagger$ Dipartimento di Produzioni Animali.

‡ Dipartimento di Scienze Ambientali. the English breeds such as the Berkshire and the Large Black. ${ }^{1}$ The Casertana (CA) and Calabrese directly descend from the Neapolitan lineage, which is formally extinct. Casertana is an endangered pig breed, very ancient and absolutely singular. It has been raised for centuries in semiwild conditions in the forests near Caserta and Benevento, where it fed on acorns, chestnuts and other vegetables of the bush.

CA and LW exhibit an opposite genetic profile with respect to the energy metabolism. Chemical composition of muscles from CA and its crossbreeds (also with LW) has already been investigated, ${ }^{9}$ underlying that CA muscles are characterized by a higher lipid content and a lower protein content. Zullo et al. reported that CA and Landrace $\times$ (Landrace $\times$ LW) pigs provided a product with the lowest chewing value; moreover, $\mathrm{CA} \times$ (Landrace $\times \mathrm{LW})$ pigs produced meat with the highest water holding capacity, while CA pigs produced lighter commercial cuts. ${ }^{9}$ Studies with CA pigs may shed light on the physiological process of fat deposition because these animals are prone to adipogenesis and have a strong aptitude for fat deposition. Indeed, CA pigs have a higher percentage of body fat and produce more than double backfat thickness as LW pigs. ${ }^{10}$ Since this breed did not undergo selection programmes, CA pigs retain the traits of a slow growing and high fat depositing pig compared to the genetic lines actually exploited in the pig industry. Comparison with LW pigs demonstrated that the CA is far less competitive regarding growth performance, reaching a commercial slaughter weight at a considerably greater age, and with a 2-fold increase in backfat thickness. ${ }^{10}$

Skeletal muscle is a heterogeneous tissue that is made up of several fiber types. ${ }^{11}$ These compositional differences deter- 